\title{
Research and Review on Novel Spread Spectrum Communication Theory
}

\author{
Wenzhun Huang \\ Department of Electronic Information Engineering \\ Xijing University \\ Xi'an, China \\ e-mail: huangwenzhun@xijing.edu.cn
}

\author{
Shanwen Zhang \\ Department of Electronic Information Engineering \\ Xijing University \\ Xi'an, China \\ e-mail: wjdw716@163.com
}

\begin{abstract}
With the rapid and bursting development of communication science and related technique, spread spectrum communication has been a hot research topic in the computer science and engineering community. Therefore, to follow the trend, in this paper, we conduct research on novel spread spectrum communication theory and corresponding anti-jamming applications. Primarily, multi-band spread spectrum communication is analyzed with theoretical analysis and de-composition, compared with conventional spread spectrum system under the condition of the same bandwidth can obtain higher information transmission rate or spread spectrum processing gain, greatly improving the utilization rate of frequency band, and its spectrum is closer to white noise, which has better resistance to intercept and confidentiality of information. We model our method through limit information transmission rate and the output signal-tonoise ratio increased with the increase of bandwidth according to the index law combined with the state-of-the-art techniques. Compared with other approach, our system outperforms and gains the better performance.
\end{abstract}

Keywords-Spread Spectrum Communication; Least Squares Estimator; Anti-jamming Applications; Communication Science; Wireless Network and Sensors

\section{INTRODUCTION}

With the rapid and bursting advancement of communication science and related techniques, the applications of the communication theory (CT) are everywhere [1]. With the development of wireless communication technology, people need more and more high accessibility and reliability to the requirement of highspeed short-range wireless communications. The emergence of UWB technology and the realization of the ultrabandwidth will support the high speed data transmission over short distances. The advantages of UWB communication technology is: high transmission rate, strong anti-interference, large system capacity, low energy, etc. Multi-band spread spectrum communication, compared with conventional spread spectrum system under the condition of the same bandwidth can obtain higher information transmission rate or spread spectrum processing gain, greatly improving the utilization rate of frequency band, and its spectrum is closer to white noise, which has better resistance to intercept and confidentiality of information. The current scholars put forward a variety of optimization analysis method, is used to filter the disturbance caused by broadband effect problem, improve the performance of
UWB technology. But most of the methods of single tone jamming circumstances only ultra-bandwidth jamming is analyzed, and the later period of ultra wideband interference under the high frequency interference analysis did not fully consider the effect of jump code, lead to the optimization of existence one-sidedness and error analysis method, cannot effectively filter interference, can effectively enhance the performance of UWB technology. Therefore, the need for enhancing the current methods is urgent.

Before analyzing the methodology proposed by us, we firstly analyze the basic applications and prior research on communication technology. In [2], Nicholas's group conducted research on the distinct effects of information technology and communication technology on firm organization. The pointed out that these have very different effects on the empowerment of employees, and by extension on wage inequality. Conversely, technologies reducing communication costs substitute agent's knowledge for directions from their managers, and lead to centralization. In [3], Barousis et al. designed and implemented the novel signal model for MIMO (Multiple-Input Multiple-Output) communication system. They find out that other architectures denoted as the single-fed MIMO system is compact parasitic array as a possible candidate to this goal. Promote the parasitic on the research and design of MIMO array applications, another signal model is put forward considering the current port array as input to the system. Based on this model, designed a series of novel. Their result is acceptable and refereed by lots of other research institutes. In [4], Ioan Toma introduced semantic technologies for scalable multi-channel communication. They present the latest developments of our multi-channel communication solution, which enables touristic providers.

Moreover, in [5], Huang's group provided insightful research on the signal subspace channel tracking for correlated underwater acoustic communication channels. They conclude that traditional least squares algorithm for channel estimation does not use the correlation structure. Research, this paper proposes a channel tracking algorithm based on model is related to the underwater acoustic communication channel. There are more related applications of communication technology in the literature reviews [6]. The applications prove that by wireless sensor networks deployed in monitoring area of a large number of sensor nodes with wireless communication function, can realize the reliable detection to the environment, including the equipment fault diagnosis. But due to some inherent 
characteristics of the network which make its facing new problems and challenges in many ways, communication interference is one of them. The presence of interference makes the receiving node to receive data needed for, thus reduces the communication efficiency of wireless sensor network. For wireless sensor network application design, reduce the interference and improve the success rate of communication is a basic problem to consider. Therefore, we conduct research on novel spread spectrum communication theory and corresponding anti-jamming applications. FH communication has been detection probability is low, the unique advantages of antiinterference ability and easy to realize multiple access, has become the importance of communication anti-jamming technology at home and abroad, is widely used in military and civilian communications. Differential frequency hopping communication system between adjacent jump signal frequency methods was used to carry information. Within each jump signal dwell time, differential frequency hopping signal modulation is a single tone, only a line on the spectrum of it. Receiver through signal processing to obtain the frequency spectrum of the received signal characteristics, according to the spectrum characteristics of the complete system synchronization and detect the current jump signal frequency, combined with the previous jump signal frequency, according to the relationship between the two signal frequency jump out information. The decisions of the receiver in the frequency domain signal frequency and therefore in the frequency domain to the differential frequency hopping system is very convenient. Our proposed method could solve the issues robustly and correctly. The experimental result proves the proposed approach gains better accuracy compared with other state-of-the-art approaches.

\section{The Theoretical Analysis of Proposed METHODOLOGY}

\section{A. The System Overview and Modelling}

In our design system, the whole work frequency band orthogonal jump frequently contains certain points. Between the sender and the receiver of $\mathrm{M}$ a channel, a channel for a jump in on one frequency point, the frequency is determined by the channel corresponding to the synchronization of frequency hopping sequence, so that the whole system needs $\mathrm{M}$ orthogonal frequency hopping sequence. Each jump on the data bits selected a channel audio signal send list. Reference signal system in classical literature the mathematical description of the form, give a binary offset carrier modulation frequency sweep the time-domain expression of PN code spread spectrum signal as the follows:

$$
\left\{\begin{array}{l}
S_{\text {sin }}=\left(\sum_{k} a_{k} \cdot \mu\left(t-k \cdot T_{c}\right) \cdot \operatorname{sgn}\left(\sin \left(2 \pi \cdot f_{s}(t) \cdot t\right)\right)\right) \\
S_{\text {cos }}=\left(\sum_{k} a_{k} \cdot \mu\left(t-k \cdot T_{c}\right) \cdot \operatorname{sgn}\left(\cos \left(2 \pi \cdot f_{s}(t) \cdot t\right)\right)\right)
\end{array}\right.
$$

MSFH also using unmodulated tone signal waveform as the air, but the data channel and compensation channel respectively according to the different frequency hopping sequence jump, reduce the probability of hit compensation channel tracking interference, weakened the tracking interference effects. MSFH, on the other hand, the multiplefrequency hopping sequence synchronized between the sender and the receiver, so the receiver RF front end can be used in a narrowband receiver, make MSFH still can "escape" partial band interference effectively. You can take some measures to the channel condition must be under the premise of increased channel capacity, also makes communication ability enhancement; Or in keeping the communication capacity under the premise of a certain, can tolerate larger noise power, also makes the anti-interference ability. Visible, channel capacity in fact shows that the communication capability of communication system, and guarantee of the ability of the communication capacity under the condition show the ability of anti-jamming. So, Shannon formula shows that the system of communication ability and anti-interference ability and transmission information of the relationship between bandwidth and signal-to-noise ratio. The formula 2 introduces the criteria.

$$
C=W \log _{2}\left[1+S /\left(n_{0} W\right)\right]
$$

In the ideal system with limit information transmission rate and the output signal-to-noise ratio increased with the increase of bandwidth according to the index law. That is, to increase bandwidth can obviously improve the output SNR. According to this principle, it should be spread signal transmission bandwidth, as much as possible in order to improve the system of the output signal-to-noise ratio, which is spread spectrum communication. The figure 1 shows the system.

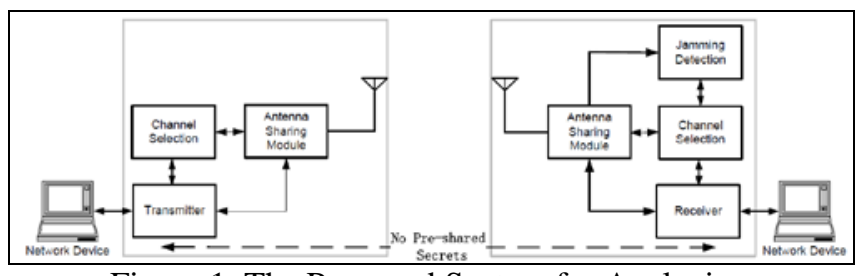

Figure 1. The Proposed System for Analyzing

It is the basic principle of direct sequence spread spectrum in the transmitting end of the communication with high-speed pseudo noise code to send information stream in spread spectrum modulation, then radio frequency modulation, make the signal in the transmission bandwidth is mainly decided by pseudo-code bandwidth, and bandwidth is greater than the original information; At the receiving end to receive radio frequency signal with local carrier for mixing, and then in the intermediate frequency with the same as the sender of pseudo-code sequences related solutions for expansion, broadband signal back into the original narrow-band signal, will be useful after demodulation regeneration unit information data recovery. Communication in the channel noise and thermal noise of background, with a very low signal power spectrum for communication, make signals submerged in noise. The following formulas describe the feature. 


$$
\begin{gathered}
\prod_{f=1}^{n} \frac{w(f) W_{t-1}\left(f+1, k-\sum_{f=1}^{f} c(f)\right)}{W_{t-1}\left(f+1, k-\sum_{f=1}^{f} c(f)\right)}=\frac{w_{i, t-1}}{W_{t-1}} \\
C_{n}\left(q, p_{J}\right)=\sum_{f \in F} q_{n}^{f} \rho_{n}^{f} C_{n}\left(q, p_{J}\right) Q_{b\left(q, P_{j}\right)}
\end{gathered}
$$

Conventional $\mathrm{FH}$ communication refers to communication both or all parties in the same synchronization algorithm and pseudo random frequency hopping pattern algorithm, under the control of the radio frequency within the frequency table in the delta pseudo random and discrete frequency synchronously jump, radio frequency in the process of jump can cover the bandwidth of the bandwidth is much larger than the original information, thus expands the spectrum. Jump when communication antijamming technology is applied in time domain. Is transmitting jump on the timeline, the timeline when can be divided into many pieces, in a frame within which the emission signal, to control by spread spectrum code sequence. Due to the simple jump anti-jamming is not strong, rarely used alone. The modelling process for the proposed approach is shown in the following formulas.

$$
\begin{aligned}
& \Delta V(t)=V(t)-V(t-T)=\int_{t-T}^{t}\left(\dot{V}_{1}+\dot{V}_{2}\right) d \tau+\Delta V_{3}(t) \leq \\
& \int_{t-T}^{t}\left(-k_{2} x^{2}-\alpha^{*} x \rho(x) \tanh \left(\frac{x \rho(x)}{\varepsilon^{j}}\right)+\tilde{\alpha} \varepsilon^{j} \hat{\alpha}+\alpha^{*}|x| \rho(x)\right) d \tau \\
& \leq \int_{t-T}^{t}\left(-k_{2} x^{2}+\left(\alpha^{*} \delta\right)+\frac{1}{4} \alpha^{* 2} \varepsilon^{j}\right) d \tau
\end{aligned}
$$$$
V(t)=V\left(t_{0}\right)+\sum_{j=0}^{i-1} \Delta V(t-j T)
$$$$
=V\left(t_{0}\right)-k_{2} \sum_{j=0}^{i-1} \int_{t-(\mathrm{j}+1) \mathrm{T}}^{t-j T} x^{2} d \tau+\left(\alpha \times \delta+\frac{1}{4} \alpha^{* 2}\right) T \sum_{i=0}^{i-1} \varepsilon^{i-j}
$$

Spread spectrum and frequency hopping technology of antijamming mechanism is different, have their own characteristics and shortcomings. In terms of strong fixed frequency interference, anti-interference is spread through the correlation algorithm to obtain the processing gain to achieve anti-jamming, but more than the fixed frequency interference tolerance will lead to a precipitous decline in spread spectrum communication interrupt or performance of the system [7]; And frequency hopping system is adopted to avoid the method of anti-interference, strong fixed frequency interference can only be one or several frequency interference frequency hopping system, will affect the performance of the system is not very serious. The detailed discussion on the theoretical parts of the method is conducted in the next section.

\section{B. The Theoretical Formulation of Our Method}

In terms of resistance to multipath interference, cannot form interference of spread spectrum system, spread spectrum system can even use the interference energy to improve system performance; To resist multipath interference and frequency hopping system, insist on every jump the dwell time is very short, not before the arrival of a receiver in multipath signal has begun to receive the nexthop signals, is heavily dependent on the device, and the ultimate jump speed problems. Direct sequence and frequency hopping hybrid spread spectrum system is on the basis of direct sequence spread spectrum systems increase the function of carrier frequency jump, the basic way of working is direct sequence spread spectrum system synchronization is based on direct sequence synchronization. When abnormal electromagnetic environment bad, or require very high anti-interference index of communication system, separate a spread spectrum system is difficult to meet the requirements, and adopt the hybrid spread spectrum system can not only meet the requirements of indicators, and easy to implement. Therefore, the hybrid spread spectrum system is very suitable for serious interference environment. The formula 7 shows the process.

$$
u=-\frac{1}{g(x)}\left(k_{2} x+f(x)+\hat{\theta} \xi(x)+\hat{\alpha} \rho(x) \tanh \left(\frac{x \rho(x)}{\varepsilon^{j}}\right)\right)
$$

Spread spectrum communication anti-jamming technology is mainly on the frequency domain by extending the communication bandwidth and reduce the signal RF general and convenient transmit power way to enhance the anti-interference ability of the communication system [8]. Rather than spread spectrum communication technology is mainly in the spatial domain, time domain, coding the antiinterference techniques of space exploration, the conventional communication system. The figure 2 shows the pattern.

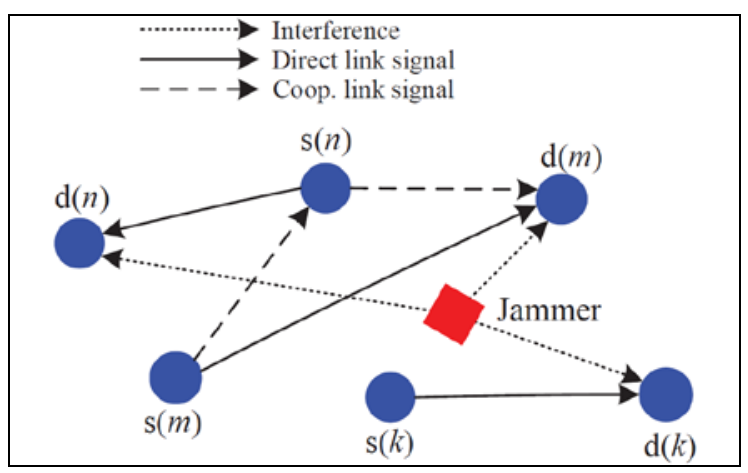

Figure 2. The Pattern for the Analyzed Graph

For various kinds of interference from different direction space, adaptive antenna can by adjusting the amplitude and phase distribution of each unit makes the lobe in the zero point is formed on the direction of interference, so as to reduce or avoid the influence of interfering signal, if the interference sources in constant motion, space adaptive antenna can change the position of the lobe zero accordingly, continue to suppress interference signals. If the jamming signal is broadband, adaptive antenna can also in the direction of the corresponding place, form a wider Angle of notch, to fight the broad band interference denoted as the equation 8 . 


$$
E\left(\left|\mathbb{N} f_{n}^{f}\right|\right)=\sum_{m \in \mathbb{N} / / n} q_{m}^{f} \Psi_{n}^{f} \sqrt{p_{i}}
$$

With digital technology and error correction coding technology to a certain extent, can improve the antijamming [9]. Error-correcting codes can correct due to the interference of random errors, mixed coding to correct the fading and interference caused by unexpected errors. The figure 3 shows the technique.

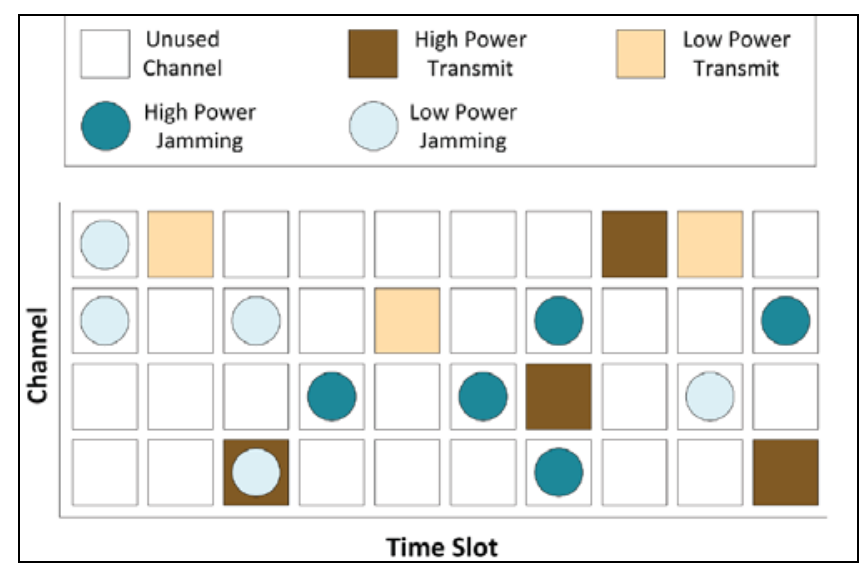

Figure 3. The Pattern for the Error Correction Model

Diversity technology includes two aspects: the content of the separation technology and combine technology. Through separation and merger, and improve the signal-to-noise ratio at the receiving end, so as to obtain diversity gain. Diversity in combating multipath transmission caused by the envelope fading and time delay, its effect is very obvious. The formula 9 illustrates the dynamic pattern.

$$
\dot{x}_{N}=f_{N}\left(x_{N}, u, \theta\right) \equiv f\left(x_{N}\right)+g\left(x_{N}\right) u+\theta^{*} \xi\left(x_{N}\right)
$$

Software radio is to use field programmable devices and modern digital signal processing technology, on the same hardware platform to achieve a variety of encoding/decoding, modulation/demodulation way, make it according to the selection of appropriate communication system, to provide effective security communication, especially with the development of microelectronics technology [10], controlled microwave, shortwave device, for the wide application of software radio technology has provided a broad prospects, as well as communication antijamming technology to create a new heaven and earth. The advanced modelling procedure is conducted as the following formulas.

$$
\begin{gathered}
|\eta(x, t)| \leq \alpha^{*} \rho(x), \forall x \in R, \forall t \in R^{+} \\
\hat{\theta}(t)=\left\{\begin{array}{cc}
\operatorname{sat}_{\bar{\theta}}(\hat{\theta}(t-T))+\gamma_{4} x \xi(x), & t \in[0, \infty) \\
0, & t \in[-T, 0)
\end{array}\right. \\
\dot{V}_{1}=x\left(-k_{2} x+\tilde{\theta}(t) \xi(x)-\hat{\alpha} \rho(x) \tanh \left(\frac{x \rho(x)}{\varepsilon^{j}}\right)+\eta(x, t)\right)= \\
-k_{2} x^{2}+\tilde{\theta}(t) \xi(x) x+x \eta(x, t)-\hat{\alpha} \rho(x) \tanh \left(\frac{x \rho(x)}{\varepsilon^{j}}\right)
\end{gathered}
$$

Track the interference by the fixed frequency communication automatic target type interference to development track the interference for frequency hopping. Improvement on tracking interference of frequency hopping communication technology dynamic mainly has three aspects [11]. As the pseudo random code is commonly used in spread spectrum communication is $\mathrm{m}$ Gold sequence and its deformation and Walsh sequences such as a few sequences, this caused some shortcomings of the spread spectrum communication can be detected. Availability of anti-jamming receiver antenna array, it is in all kinds of interference environment the probability that the receiver can work. Signal, the interference may come from all directions, so the signal and the direction of interference can be thought of as a random variable, thus available function $\mathrm{C}$ is also a random variable. Using Benoit the assumption for the analysis of single signal availability and the relationship between the signal that are available. In fact, is decided by the navigation constellation configuration, is the existence of spatial correlation between each satellite. Especially for static applications such as monitoring receiver, due to the receiving antenna array position and posture are unchanged, navigation signals relative to the incident direction of the plane array depends entirely on the constellation characteristics with the receiver to the location. So looking for new and better spread spectrum code sequence is an important aspect in the development of communication anti-jamming technology, improve the antijamming ability of spread spectrum communication is important research direction. In order to improve the antiinterference ability, usually need to take correction, interweave, encryption and other measures, these measures will reduce the effective information transmission rate, therefore, at the same time of improving frequency hopping rate, also need to increase the signal bandwidth, thus suppressing interference and improve the transmission rate of dual purpose.

In such a complex electromagnetic environment, to play the advantages of short wave communication, the rational allocation of shortwave communication resources, establish a composite system of multi-level and multiple frequency, multi-channel, routing and link of short-wave communication network system, through the anti-jamming equipment of network, comprehensive utilization of all kinds of resources in the network system, the efficient use of network information, jamming methods for planning and configuration, effectively improve the anti-jamming ability of the system. In the next section, we will conduct experiment on the system.

\section{EXPERIMENT AND SIMULATION RESULT}

In this section, the performance of the proposed methodology is evaluated. A few test validated the theory expression and statistical model of assumptions (in the previous section) asymptotic properties of the interpolation method is proposed. Simulation scenario to consider our work refers to satellite DS/SS CDMA communication, especially in communication (the next) European Galileo scenario. Algorithm on different tap several polynomial 
capture performance. With 21 order polynomial respectively 2 and 3 to generate polynomial polynomials, the tap number 5 and 11, respectively, in different SNR conditions are simulated. Ideally, when receiving sequence is long enough, caught algorithm performance and the polynomial order only, has nothing to do with the number of tap. The figure 3 and figure 4 show the result for the experiment sets.

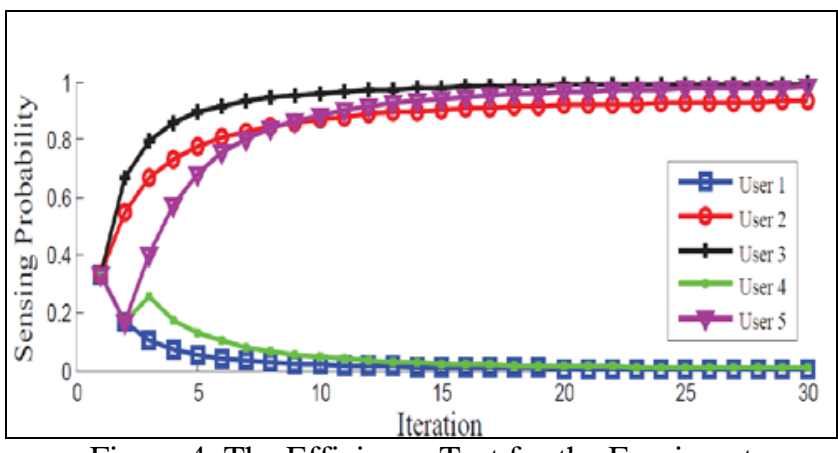

Figure 4. The Efficiency Test for the Eperiment

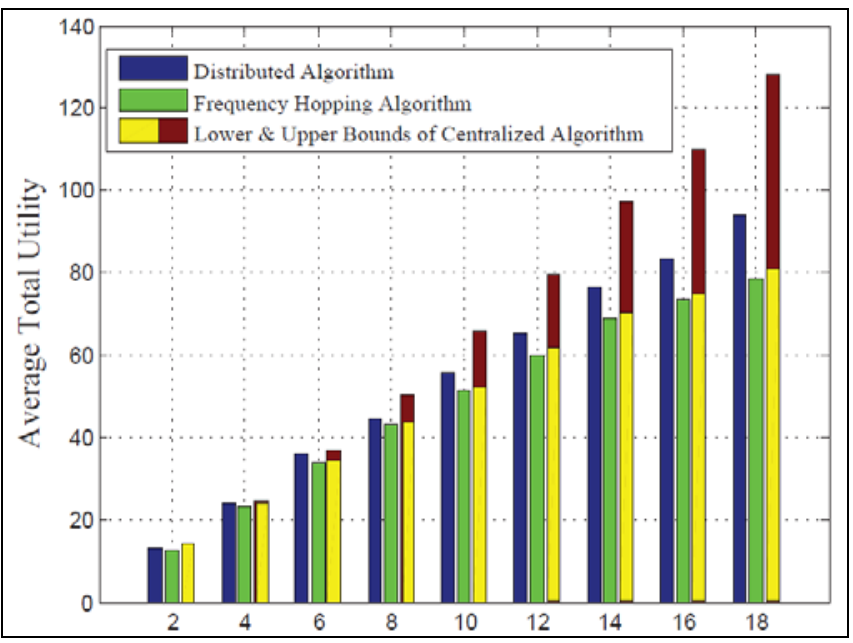

Figure 5. The Comparision Experiment fo the System

\section{CONCLUSION AND SUMMARY}

In this paper, we conduct research on novel spread spectrum communication theory and corresponding antijamming applications. Multi-band spread spectrum communication, compared with conventional spread spectrum system under the condition of the same bandwidth can obtain higher information transmission rate or spread spectrum processing gain, greatly improving the utilization rate of frequency band, and its spectrum is closer to white noise, which has better resistance to intercept and confidentiality of information. The current scholars put forward a variety of optimization analysis method, is used to filter the disturbance caused by broadband effect problem, improve the performance of UWB technology. As the pseudo random code is commonly used in spread spectrum communication is $\mathrm{m}$ Gold sequence and its deformation and Walsh sequences such as a few sequences, this caused some shortcomings of the spread spectrum communication can be detected. The experiment result shows the feasibility of our method, in the future, we plan to combine the optimization technique to polish the current methodology.

\section{ACKNOWLEDGMENT}

This work is financially supported by the scientific research program of Shaanxi Provincial Education Department (Program No. 14JK2156).

\section{REFERENCES}

[1] Xie, Xinqian, Mugen Peng, Bin Zhao, Wenbo Wang, and Yingbo Hua. "Maximum a posteriori based channel estimation strategy for twoway relaying channels." Wireless Communications, IEEE Transactions on 13, no. 1, 2014, pp. 450-463.

[2] Bloom, Nicholas, Luis Garicano, Raffaella Sadun, and John Van Reenen. "The distinct effects of information technology and communication technology on firm organization." Management Science 60, no. 12, 2014, pp. 2859-2885.

[3] Barousis, Vlasis I., Constantinos B. Papadias, and Ralf R. Muller. "A new signal model for MIMO communication with compact parasitic arrays." In Communications, Control and Signal Processing (ISCCSP), 2014 6th International Symposium on, pp. 109-113. IEEE, 2014.

[4] Toma, Ioan, Christoph Fuchs, Corneliu Stanciu, and Dieter Fensel. "Using semantic technologies for scalable multi-channel communication." In The Semantic Web: ESWC 2014 Satellite Events, pp. 516-520. Springer International Publishing, 2014.

[5] Huang, S. H., Jenho Tsao, T. C. Yang, and Sheng-Wen Cheng. "Model-based signal subspace channel tracking for correlated underwater acoustic communication channels." Oceanic Engineering, IEEE Journal of 39, no. 2 (2014): 343-356.

[6] Huang, S. H., Jenho Tsao, T. C. Yang, and Sheng-Wen Cheng. "Model-based signal subspace channel tracking for correlated underwater acoustic communication channels." Oceanic Engineering, IEEE Journal of 39, no. 2, 2014, pp. 343-356.

[7] Kanungo, Guru Kalyan, et al. "Mammogram Image Segmentation Using Hybridization of Fuzzy Clustering and Optimization Algorithms." Intelligent Computing, Communication and Devices. Springer India, 2015, pp. 403-413.

[8] Mosalaosi, M., and Thomas JO Afullo. "Broadband Analysis and Characterization of Noise for In-door Power-line Communication Channels." In PIERS Proceedings. 2014.

[9] Ahmed, Salman, Feng Xiao, and Tongwen Chen. "Asynchronous consensus-based time synchronisation in wireless sensor networks using unreliable communication links." IET Control Theory \& Applications 8, no. 12, 2014, pp. 1083-1090.

[10] Brassard, Gilles, Ashwin Nayak, Alain Tapp, Dave Touchette, and Falk Unger. "Noisy interactive quantum communication." In Foundations of Computer Science (FOCS), 2014 IEEE 55th Annual Symposium on, pp. 296-305. IEEE, 2014.

[11] Giovannetti, V., Raúl García-Patrón, N. J. Cerf, and A. S. Holevo. "Ultimate classical communication rates of quantum optical channels." Nature Photonics 8, no. 10 (2014): 796-800. 